\title{
Maternal Hypotension
}

National Cancer Institute

\section{Source}

National Cancer Institute. Maternal Hypotension. NCI Thesaurus. Code C92829.

A symptomatic decrease in baseline systolic or diastolic blood pressure in a pregnant woman that requires intervention. 